\title{
Note du Rédacteur en chef
}

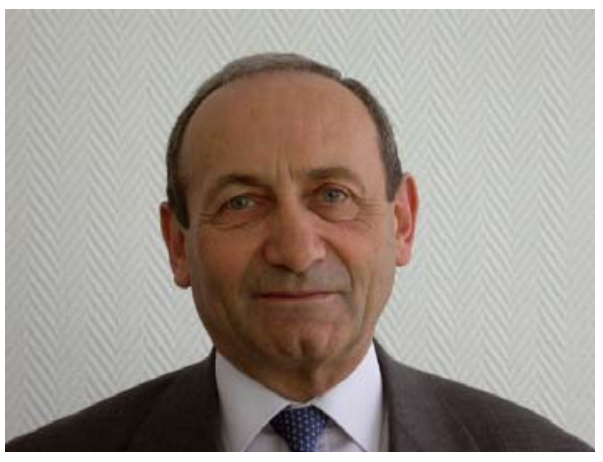

En un temps où les nouvelles technologies impriment une forte impulsion à la technique, où les notions de développement durable et de respect de l'environnement imposent de nouvelles exigences, où les aspects sécurité et sanitaires prennent de plus en plus d'importance, les matériaux et leurs méthodes d'élaboration apparaissent plus que jamais incontournables.

Plusieurs éléments ont conduit à cette situation qui s'impose actuellement avec force :

- Les progrès dans les connaissances scientifiques, des méthodes de simulation et de modélisation, ont permis la conception de nouveaux matériaux dont les propriétés ont ensuite pu être exploitées pour résoudre de nouveaux problèmes : semiconducteurs, quasicristaux, alliages à mémoire de forme, matériaux adaptatifs.. .

- D'autres matériaux ont été développés spécifiquement pour répondre à de nouveaux besoins : composites céramiques, fibres textiles, matériaux à gradients, matériaux à structure désordonnée, matériaux nanostructurés...

- Parallèlement, les aspects développement durable ont mis en avant les notions d'économie d'énergie lors de la mise en œuvre et l'utilisation, et de recyclabilité.

Il en a résulté pour les matériaux de devoir répondre à de nouveaux besoins et de présenter des qualités qui jusqu'à ces derniers temps n'étaient pas suffisamment prises en compte. Cela a donc imposé une maîtrise accrue de l'élaboration, des propriétés et de la tenue en service en relation avec la structure.

Matériaux \& Techniques a souhaité s'ouvrir à ces différents aspects. De nouvelles sections ont doncété ouvertes concernant les nouveaux matériaux, les méthodes d'élaboration et la tenue en service, leur modélisation et leur simulation, qui prennent en compte des sujets tels que :

- Matériaux à structure désordonnée ;

- Matériaux nanostructurés ;

- Recyclabilité.

Ce numéro thématique sur les «verres » en est un premier exemple qui réunit plusieurs articles concernant les verres et vitrocéramiques, leur propriété, leur tenue en service, des méthodes et techniques de contrôle et analyse, des exemples d'application.

René Gras

Rédacteur en chef de Matériaux \& Techniques 